\title{
Confined Enzymatic Activity of S. scitamineum and U. esculenta at the Smut Gall during Infection
}

\author{
Robinson C Jose ${ }^{1-3}$, Grihalakshmi D Nongthombam ${ }^{1-3}$, Bengyella Louis ${ }^{4}$, Pratap J Handique ${ }^{2}$ and Narayan C Talukdar ${ }^{3^{*}}$ \\ ${ }^{1}$ Institute of Bioresources and Sustainable Development (IBSD), Takyelpat, Imphal-795001, Manipur, India \\ ${ }^{2}$ Department of Biotechnology, Guwahati University, Guwahati-781014, Assam, India \\ ${ }^{3}$ Division of Life Sciences, Institute of Advanced Study in Science and Technology, Guwahati 781035, Assam, India \\ ${ }^{4}$ Department of Biotechnology, Faculty of Applied and Computer Sciences, Vaal University of Technology, South Africa
}

*Corresponding author: Talukdar NC, Division of Life Sciences, Institute of Advanced Study in Science and Technology, Guwahati 781035 , Assam, India, Tel: +61 8 8313 6738; E-mail: nctalukdar@yahoo.com

Received date: March 23, 2017; Accepted date: June 26, 2017; Published date: July 4, 2017

Copyright: (C) 2017 Jose RC, et al. This is an open-access article distributed under the terms of the Creative Commons Attribution License, which permits unrestricted use, distribution and reproduction in any medium, provided the original author and source are credited.

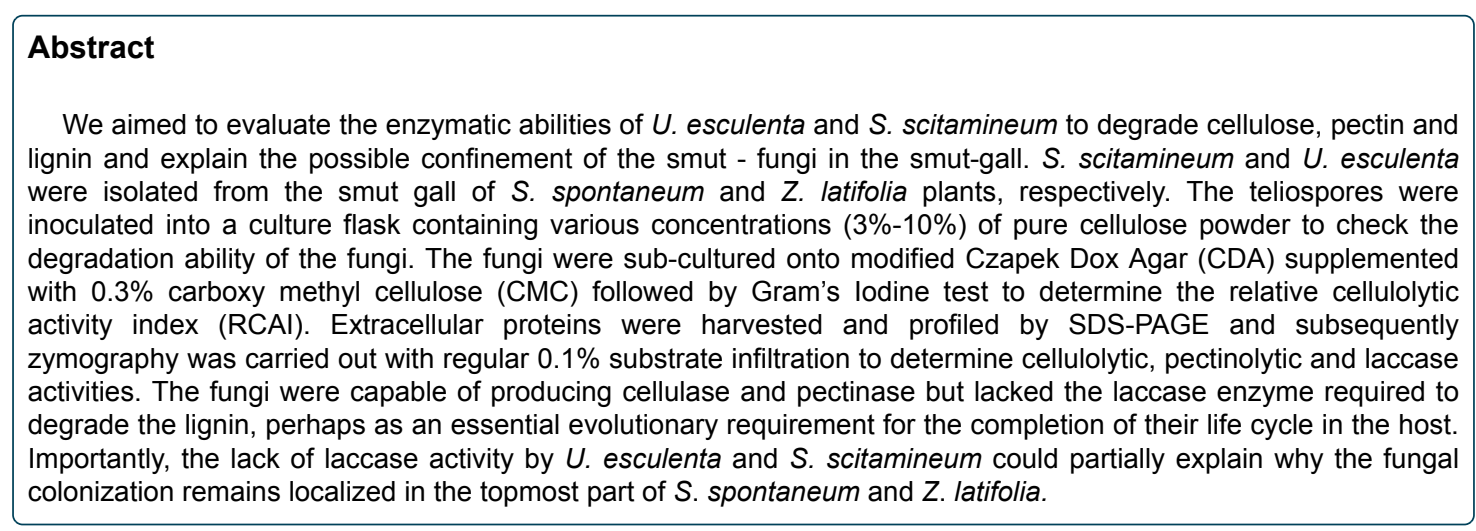

Keywords: Smut; Enzyme; SDS-PAGE; Zymogram; PCW

\section{Introduction}

Cellulose, hemicellulose, pectin, and lignin are the major components of plant cell wall [1]. The organization and interactions of cell wall components are still not known with certainty and there is still considerable debate about how cell wall organization is modified to allow cells to expand and grow particularly in the presence of biotrophic fungal endophytes. Cellulase, pectinase and ligninase are produced extracellularly by most microbes and play a significant role in degrading various polysaccharides to produce glucose for their growth and survival $[2,3]$.

It is a well-known fact that the living organisms try to adapt themselves with the environment by adopting different strategies to the prevailing conditions. Based on field observations and data generated in the Indo-Burma biodiversity hotspot on $S$. scitamineum and $U$. esculenta [4], it is thought that smut fungi often undergo unique adaptation in their biota, where they evolved to become associated with various plants of the family Poaceae.

Zizania latifolia is a wild rice which does not give out rice grains due to the presence of a fungal endophyte $U$. esculenta [4-6] restricted to few selected habitats of South East Asia [7]. Strangely, occurrence of $Z$. latifolia in India is found to confine only in the swampy habitats and wetlands of Imphal valley, Manipur [8]. Similarly, S. scitamineum is another smut fungus that harbors inside Saccharum spontaneum and is also found in the Indo-Burma biodiversity hotspot of India [8]. The prevalence of $S$. scitamineum in the field results in the swelling of the upper parts of $S$. spontaneum in the form of smut. The infection of both $U$. esculenta and $S$. scitamineum interferes with inflorescence and seed production by their respective host $[4,5]$.

The fungi $S$. scitamineum and $U$. esculenta often form localized infection in $S$. spontaneum and $Z$. latifolia during biotrophic interactions that develops into smut-gall. Advanced microscopic studies revealed that these fungi do not degrade the sclerenchymatous cells found in the vascular bundles. It is not clear whether these fungi lacks cellulolytic, pectinolytic and laccase activities to invade their host systemically and thus confine only to the smut-gall portion of the infected plant. In this present study, we determined the cellulolytic/ pectinolytic/laccase activities of $S$. scitamineum and $U$. esculenta and correlate the differences in their enzymatic potential with evolutionary divergence.

\section{Materials and Methods}

\section{Microorganism}

The fungi $S$. scitamineum (Genbank accession gi|744827319|gb| KP068082.1) and $U$. esculenta (Genbank accession gi|643431282|gb| KJ627848.1) were isolated from the smut gall of $S$. spontaneum and $Z$. latifolia, respectively and were used in this study.

\section{Fluorescence microscopy}

The distribution and the area colonized by fungus inside the plant after the formation of smut gall were studied by fluorescence microscopy. Tissue sections of thickness $10-250 \mu \mathrm{m}$ from smut-gall 
were generated by using Leica CM-3050S cryostat microtome. The sections containing $U$. esculenta and the interaction zones were stained with $100 \% \mathrm{v} / \mathrm{v}$ calcofluor-white stain (CWS; Sigma ${ }^{\circ}$, Saint Louis, USA) that binds to the cellulose materials and was performed according to the manufacturer's instructions. All the observations were carried out by fluorescent Olympus BX61 microscope fitted with fluorescent filters, coupled with image pro plus 5.1.

\section{Screening for cellulase activity}

The fungus was grown on modified Czapak Dox Agar (CDA) medium consisting of the following $(g L-1)$ : $\mathrm{NaNO}_{3}, 2.0 ; \mathrm{K}_{2} \mathrm{HPO}_{4}, 1.0$; $\mathrm{NaCl}, 0.5 ; \mathrm{MgSO}_{4} .7 \mathrm{H}_{2} \mathrm{O}, 0.5 ; \mathrm{FeSO}_{4} .7 \mathrm{H}_{2} \mathrm{O}$ 0.001; supplemented with $0.3 \%$ carboxy methyl cellulose (CMC). After 2 days of incubation at $20^{\circ} \mathrm{C}$ and $30^{\circ} \mathrm{C}$, the culture plates were flooded with Gram's Iodine for 2-3 min. Positive activity was determined based on the relative cellulolytic activity index (RCAI).

\section{Cellulose degradation}

Teliospores of $S$. scitamineum and $U$. esculenta were inoculated into a culture flask containing various concentrations (3\%-10\%) of the pure cellulose powder along with control flask with no inoculation. Fluorescent microscopic images of the Cellulose powder (Sigma', Saint Louis, USA) with different inoculated strains were stained with CWS after 21 days of stationary incubation at $30^{\circ} \mathrm{C}$.

\section{Protein quantification}

The protein content was estimated using the dye-binding method [9]. $15 \mu$ protein sample was mixed with $500 \mu \mathrm{l}$ of Bradford reagent (five diluted) (Bio-rad" Laboratories, Hercules, CA, USA) and allowed for $15 \mathrm{~min}$. The absorbance was taken spectrophotometrically at 595 $\mathrm{nm}$. Bovine serum albumin (BSA) dissolved in ReadyPrep rehydration buffer was used for establishing the standard curve.

\section{Profiling extracellular proteins}

Extracellular proteins from culture supernatant precipitated using $\left(\mathrm{NH}_{4}\right)_{2} \mathrm{SO}_{4}$ was used to study the protein profile. Sodium dodecyl sulfate polyacrylamide gel electrophoresis (SDS-PAGE) was performed in $5 / 12 \%$ and $5 / 14 \%$ gels.

To determine the protein bands with cellulolytic, pectinolytic and laccase activity, regular SDS-PAGE was performed with $0.1 \%$ substrate infiltration but avoiding boiling and $\beta$-mercaptoethanol to the sample buffer. After the run, gels were incubated in 1\% Triton-X-100 for 30 min to remove SDS (repeated twice). The gel was then washed extensively in distilled water to remove the excess Triton-X-100 and then again incubated for $1 \mathrm{~h}$ at $50^{\circ} \mathrm{C}$ in $50 \mathrm{mM}$ sodium citrate buffer at the $\mathrm{pH}$ optimum of each enzyme [10].

\section{Phylogenetic analysis}

Phylogenetic analysis was performed based on rDNA sequences for $U$. esculenta and $S$. scitamineum. Sequence alignment was performed using Muscle program. Best substitution model parameters were determined based on Akaike information criterion, corrected (AICc) and Bayesian information criterion (BIC). The evolutionary history was inferred using the maximum likelihood (ML) method and rooted as previously described [11] and all the analysis were performed in MEGA 6 software [12]. The strength of the internal branches of the ML tree was statistically tested by performing 1000 bootstrap replications.

\section{Results and Discussion}

This study shows the ability of two smut fungi to produce cellulase, pectinase and the evidence explaining the need of various enzyme secretions in varying amounts depending upon different circumstances a fungus encounters during its growth in vivo and in vitro. It was noted that $S$. scitamineum (herein referred as gi|744827319) and U. esculenta (herein referred as gi|643431282) isolated from the smut gall of $S$. spontaneum and $Z$. latifolia, respectively (Figures 1 and 2), produced white mats on PDA plates after 14 days of growth (Figure 3).

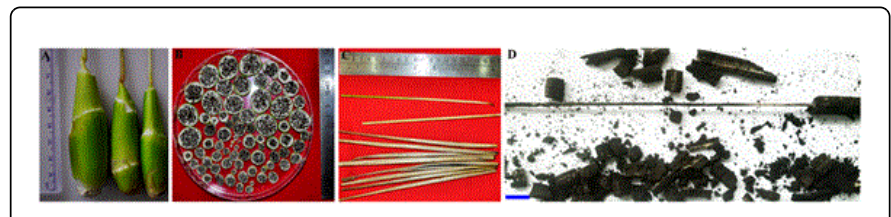

Figure 1: Morphological features of the smut galls on Zizania latifolia and Saccharum spontaneum. A-B Morphology of the $Z$. latifolia smut gall after $U$. esculenta infection and its horizontal cross sections. C-D Morphology of the $S$. spontaneum smut gall after $S$. scitamineum infection and the longitudinal inner view of smut, Bar $=0.5 \mathrm{~cm}$.

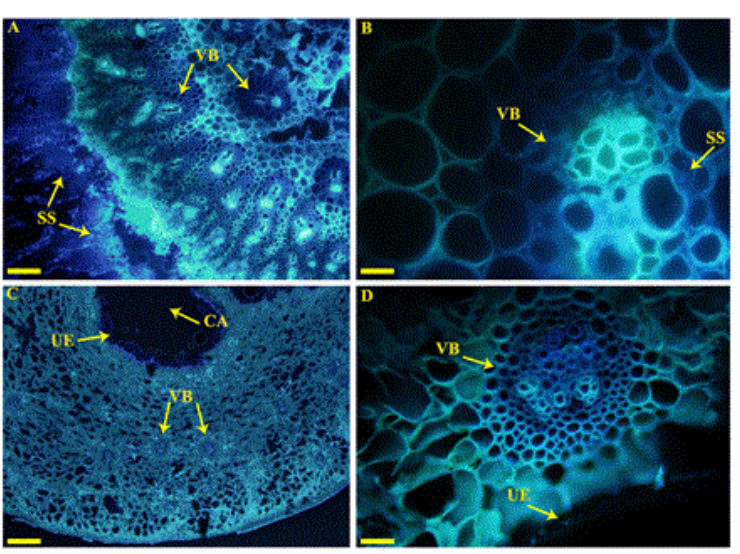

Figure 2: Fluorescent microscopic images of the cross sections, showing the vascular bundle of the infected $S$. spontaneum stalk with $S$. scitamineum and that of $Z$. latifolia with $U$. esculenta stained with CWS. A-B, horizontal cross sections showing the intact vascular bundle (VB) and the fungal material of $S$. scitamineum (SS) mainly teliospores and sporidia and hyphae on the peripheral epidermal and inner cortical cells (CC), scale bar $A=200 \mu \mathrm{m}$ and $\mathrm{B}=50 \mu \mathrm{m}$. C-D, Horizontal cross sections showing the intact vascular bundle (VB), and the fungal material of $U$. esculenta (UE) mainly teliospores, with the formation of cavity (CA) scale bar $A=500 \mu \mathrm{m}$ and $\mathrm{B}=25 \mu \mathrm{m}$. 

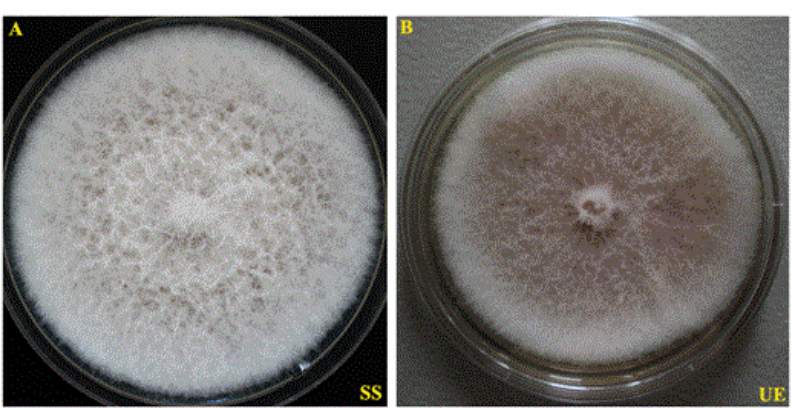

Figure 3: Colony feature of Sporisorium scitamineum (SS) and Ustilago esculenta (UE) fungal isolate from the smut gall of $S$. spontaneum and Zizania latifolia respectively grown in PDA plate for 14 days containing chloramphenicol at the concentration of 25 $\mathrm{mg} \mathrm{L}^{-1}$.

These cultures were subsequently subcultured and used for studying their cellulolytic, pectinolytic and ligninase activity. Fluorescent microscopic images of the cellulose powder (Sigma , Saint Louis, USA) were stained with CWS after 14 days of inoculation with different fungi. It was found that the smut fungi converted cellulose powder into small subunits compared to uninoculated control (Supplementary Figure S1). The $S$. scitamineum was able to carry out more breakdown of the cellulose compared to U. esculenta from the microscopic images obtained (Supplementary Figure S1). In this study, we found that the cellulose breakdown capabilities of the two smut fungi and zymogram assays further confirmed that $S$. scitamineum had better abilities compared to $U$. esculenta as evident from the relative clearance zone formed (Supplementary Figure S2). The extracellular protein profiles of $S$. scitamineum and $U$. esculenta obtained on $12 \%$ gel showed distinct bands of cellulase and pectinase. It was found that $S$. scitamineum and $U$. esculenta differentially secreted extracellular proteins (Supplementary Figures S3-S6 and Figure 4).

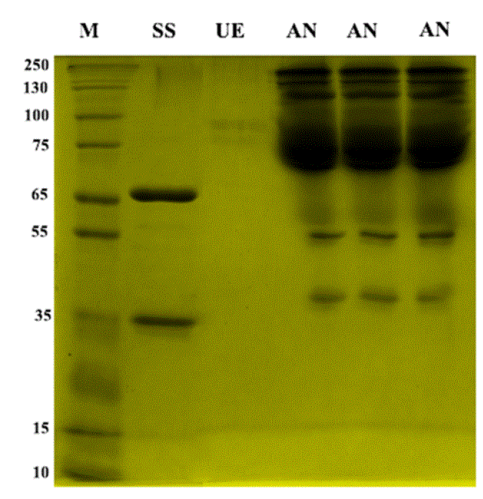

Figure 4: SDS-PAGE Gel of $12 \%$ showing the extracellular protein profile of the fungus Sporisorium scitamineum (SS) and Ustilago esculenta (UE) along with the positive control Aspergillus niger (AN). Note; $\mathrm{M}$ is the molecular weight marker in $\mathrm{kDa}$.

The smut fungi extracellular proteins did not show any activity on the zymogram when lignin was used as a supplement in the gel.
On the basis of the divergent results observed on the enzymatic activity, we generated the phylogenetic history for the two species (Figure 5). The best substitution method was $\mathrm{K} 2+\mathrm{I}$ [13] based on $\mathrm{BIC}=2560.54$ and $\mathrm{AICc}=2178.73$. The rate variation model allowed for some sites to be evolutionarily invariable $([+\mathrm{I}], 76.6839 \%$ sites $)$. The transversion/transition rates were $[C \leftrightarrow G]=0.06, \quad[A \leftrightarrow G]=0.14$, $[\mathrm{T} \rightarrow \mathrm{G}]=0.06,[\mathrm{~T} \rightarrow \mathrm{C}]=0.14,[\mathrm{C} \rightarrow \mathrm{A}]=0.06$ and the estimated transition/ transversion bias was 1.27 . Within the dataset, 325 patterns were found. Out of a total of 1450 sites, 1242 sites were without polymorphism $(85.66 \%)$. The tree was generated at a highest log likelihood of -1032.53 (Figure 5). Two distinct clades (I and II) were formed and the overall distance (d) among taxa was 0.057 . The studied species were distant from each other, reaffirming the disparity in their enzymatic profile although both form smut-gall in the same ecosystem. Thus, indicating that, they must have evolved differently yet conserve the traits to cause localized infection in their host plants.

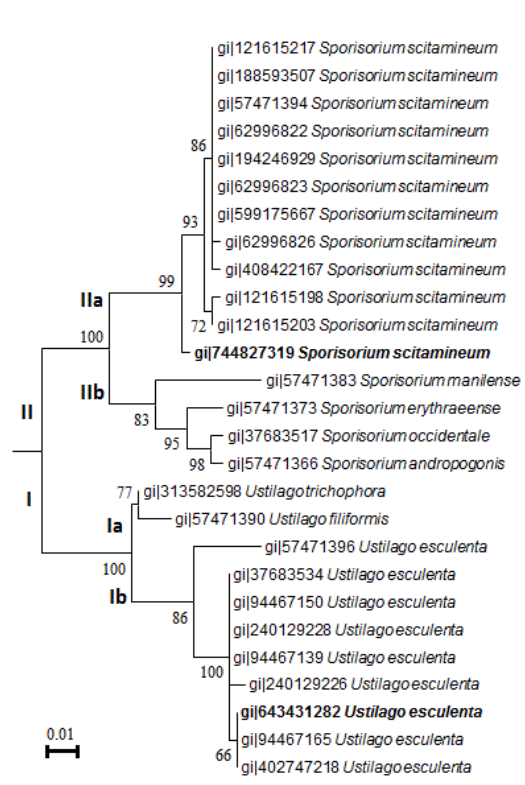

Figure 5: Molecular phylogenetic analysis by the maximum likelihood method. The percentage of trees in which the associated taxa clustered together is shown next to the branches. The tree is drawn to scale, with branch lengths measured in the number of substitutions per site.

Previous study showed that fungi flagellum was lost early during evolution; thus, the majority of fungi species lacks flagellum [14]. By default, absence of flagellum could have prompted some fungi to depend on their enzymatic potential to explore their immediate niche or associate with non-selective hosts to derive their food, protection and other conditions to complete their life cycle. This might have encouraged certain fungi to modulate its enzyme secretions to find a strategic host without harming it drastically. A fungus generally secretes less amount of enzyme while establishing a biotrophic relationship with the plant as found in $U$. maydis - Z. mays interaction [15].

Because of the absence of fungal material inside the vascular bundles of the smut galls and the presence of hyphae in the intercellular spaces of nearby ground tissue in $Z$. latifolia and $S$. spontaneum [8], two hypotheses could be proposed. Firstly, the 
observation could be due to the fact that smut fungi are naturally incapable of producing the laccase enzyme required to degrade the sclerenchymatous cells of the vascular bundle containing significant amount of lignin. Secondly, the plant could mount counter defence mechanism aimed at suppressing the fungal activity. We observed that when the fungi are taken out of the host and deprived of their natural source of food, triggers in them the trait to express various enzymes. Hence, the smut fungi when grown on media devoid of any plant material showed more cellulase and pectinase enzymes which we believe were suppressed previously by the plant's defense system and/or due to easy availability of nutrition.

After 2 days of incubation at $20^{\circ} \mathrm{C}$ and $30^{\circ} \mathrm{C}$, the culture plates of $U$. esculenta and S. scitamineum flooded with Gram's Iodine for 2-3 min showed positive cellulolytic activity in the form of clear zones. The cellulolytic and pectinolytic activity of $S$. scitamineum and $U$. esculenta were further confirmed with the bands on SDS-PAGE along with a positive control of extracellular protein of Aspergillus niger and further by a zymogram assay. This study reveals that smut fungi generally does not produce cellulase and pectinase and other PCW (Plant Cell Wall) degrading enzymes when inside or on the plant at significant levels so as to evade plants counter defense system.

Our findings confirm that smut fungi are naturally incapable of degrading the lignin material, while it has the ability to degrade the cellulose and pectin material of the plant and complies with microscopic and zymographic results (Supplementary Figures S5 and S6). The fungi $U$. esculenta and $S$. scitamineum failed to produce any clear band in the zymogram when lignin was supplemented to the gel and validated the inference derived from the in vivo microscopic observations. It was observed that the lipid content was higher in $U$. esculenta than the $S$. scitamineum. This could be one of the reasons which complemented the energy requirements of $U$. esculenta whereas $S$. scitamineum had to depend more on its enzymatic activity for the energy (Supplementary Figure S7).

The cellulose and hemicellulose components of the plant cell walls are intimately associated with the lignin moiety in most cases and consequently may present a barrier to the hydrolytic enzymes catalyzing the degradation of the polysaccharide. Although the cellulolytic and pectinolytic capacity of smut fungi is clearly evident from this study the fungi aversion for lignified substrate unquestionably revealed their apparent inability to synthesize any of the recognized lignin-transforming enzymes in the plant as well as in the media.

Lignin has always been associated with reduced digestibility of the overall plant biomass, which helps defend against pathogens and pests [16]. Since smut fungi appear to lack a lignin lytic or lignin transforming system, we suggest they could have been evolved for the proper incubation time required for the fungus on the plant to complete their life cycle without killing the plants prematurely prior to inflorescence. Thereby accumulating sufficient nutrition for the next generation and ensuring progeny existence and spread of smut fungi.

Phenotypically, the studied fungi form smut-gall but genetically placed in different clades and sub clades (Figure 5). Although they have evolved differently based on rDNA analysis, they have conserved similar enzymatic potentials to cause localized infection that form smut-gall. Interestingly, neither $S$. scitamineum nor $U$. esculenta clustered with other taxa in subclade IIa and IIb, respectively, suggesting that the smut fungi have evolved differently. It is now accepted that fungi evolution has been successful since they diverged from other life around 1,500 million years ago [17], with the glomaleans branching from the "higher fungi" at $~ 570$ million years ago [18], providing clues that the process is dynamic. It could be interesting to mine the signalling molecules exchanged between the fungi and the host in order to affirm whether this rDNA divergence is maintained at the chemical level [19].

\section{Conclusion}

This study reveals the cellulase and pectinase activity of the smut fungi $U$. esculenta and $S$. scitamineum and stressed on the lack of ligninase activity and the possible evolutionary background to this phenomenon. It was found that the two smut fungi produce more cellulase at $30^{\circ} \mathrm{C}$ than at $20^{\circ} \mathrm{C}$. The various smut fungi found on various hosts can be assumed to be a potential candidates for the cellulase and pectinase enzymes. It is proposed that, confine development of smut-gall at the topmost part of $S$. spontaneum and $Z$. latifolia could be due to lack of ligninase activities by $S$. scitamineum and $U$. esculenta preventing systemic colonization of their host.

\section{Acknowledgement}

This work was supported by DST-INSPIRE as fellowship (No: IF120609). Our sincere thanks go to the lab members of CMBL and MRD in IBSD, DBT, Imphal for their support and encouragement.

\section{Conflict of Interest}

The authors declare no conflict of interest.

\section{References}

1. Bacic A, Harris, PJ, Stone BA (1988) Structure and function of plant cell walls. The Biochemistry of plants 14: 297-331.

2. Gueguen Y, Chemardin P, Janbon G, Arnaud A, and Galzy P (1996) A very efficient $\mathrm{B}$-glucosidase catalyst for the hydrolysis of flavor precursors of wines and fruit juices. J Agri Food Chem 44: 2336-2340.

3. Bhatia Y, Mishra S, Bisaria VS (2002) Microbial B-glucosidases: Cloning, properties and applications. Crit Rev Biotechnol 22: 375-407.

4. Jose RC, Goyari S, Louis B, Waikhom SD, Handique PJ, et al. (2016) Investigation on the biotrophic interaction of Ustilago esculenta on Zizania latifolia found in the Indo-Burma biodiversity hotspot. Microb Pathog 98: 6-15.

5. Chan TS, Thrower LB (1980) The host- parasite relationship between Zizania caduciflora Turcz and Ustilago esculenta P. Henn. I. Structure and development of the host and host parasite combination. New Phytol. 85: 201-207.

6. Yang HC, Leu LS (1978) Formation and histopathology of galls induced by Ustilago esculenta in Zizania latifolia. Phytopathology 68: 1572-1576.

7. Xu X, Walters C, Antolin MF (2010) Phylogeny and biogeography of the eastern Asian-North American disjunct wild-rice genus (Zizania L. Poaceae). Mol Phylogenetics Evol 55: 1008-1017.

8. Jose RC, Louis B, Goyari S, Waikhom SD, Handique PJ, et al. (2016) Biotrophic interaction of Sporisorium scitamineum on a new hostSaccharum spontaneum. Micron 81: 8-15.

9. Karnchanatat A, Petsom A, Sangvanich P, Piapukiew J, Whalley AJS, et al. (2008) A novel thermostable endoglucanase from the wood-decaying fungus Daldinia eschscholzii (Ehrenb.:Fr.) Rehm. Enzyme Microb Technol 42: 404-413.

10. Louis B, Sayanika DW, Pranab R, Pardeep KB, Wakambam MS, et al. (2014) Host shifting dynamics of Cochliobolus lunatus. From a biocontrol agent to a severe environmental threat. Biomed Res Int, pp: $1-10$. 
Citation: Jose RC, Grihalakshmi ND, Louis B, Handique PJ, Talukdar NC (2017) Confined Enzymatic Activity of S. scitamineum and U. esculenta at the Smut Gall during Infection. Biol Syst Open Access 6: 182. doi:10.4172/2329-6577.1000182

Page 5 of 5

11. Tamura K, Stecher G, Peterson D, Filipski A, Kumar S (2013) MEGA6: Molecular evolutionary genetics analysis version 6.0. Mol Biol Evol 30: 2725-2729.

12. Tamura K, Nei M (1993) Estimation of the number of nucleotide substitutions in the control region of mitochondrial DNA in humans and chimpanzees. Mol Biol Evol 10: 512-526.

13. Liu YJ, Hodson MC, Hall BD (2006) Loss of the flagellum happened only once in the fungal lineage: Phylogenetic structure of Kingdom Fung inferred from RNA polymerase II subunit genes. BMC Evol Biol 6: 74.

14. Sarkanen KV, Ludwig CH (1971) Lignins: Occurrence, formation, structure and reactions. Wiley Intersci, New York.

15. Kamper J, Kahmann R., Bolker M., MaL J, Brefort T. (2006) Insights from the genome of the biotrophic fungal plant pathogen Ustilago maydis. Nature 444: 97-101
16. Wang DC, Kumar S, Hedges SB (1999) Divergence time estimates for the early history of animal phyla and the origin of plants, animals and fungi. Proc Biol Sci 266: 163-171.

17. Tehler A, Little DP, Farris JS (2003) The full-length phylogenetic tree from 1551 ribosomal sequences of chitinous fungi, Fungi. Mycol Res 107: 901-916.

18. Zeng F, Zhang Y, Zhang Z, Malik A, Lin Y, (2017) Multiple-site fragment deletion, insertion and substitution mutagenesis by modified overlap extension PCR. Biotechnol Biotechnol Equip 31: 339-348.

19. Bradford M (1976) A rapid and sensitive method for the quantitation of microgram quantities of protein utilizing the principle of protein-dye binding. Anal Biochem 72: 248-254. 